\title{
Coherent control and giant enhancement of multiphoton ionization and high-order-harmonic generation driven by intense frequency-comb laser fields: An ab initio theoretical investigation
}

\author{
Di Zhao, ${ }^{1,2} \mathrm{Fu}-\mathrm{li} \mathrm{Li},{ }^{1}$ and Shih-I Chu ${ }^{2, *}$ \\ ${ }^{1}$ Department of Applied Physics, Xi'an Jiaotong University, Xi'an 710049, China \\ ${ }^{2}$ Department of Chemistry, University of Kansas, Lawrence, Kansas 66045, USA
}

(Received 25 December 2012; published 11 April 2013)

\begin{abstract}
We present an $a b$ initio theoretical investigation of the coherent control and significant enhancement of multiphoton ionization (MPI) and high-order-harmonic generation (HHG) of atoms and molecules by means of intense frequency-comb laser fields. We show that the nonperiodically or quasiperiodically time-dependent Schrödinger equation for the frequency-comb laser excitation problem can be transformed exactly into a timeindependent non-Hermitian generalized Floquet matrix eigenvalue problem by means of the many-mode Floquet theory and the complex-scaling transformation. The generalized Floquet Hamiltonian can be optimally discretized and the complex quasienergy eigenvalues and eigenfunctions can be solved accurately and efficiently by means of the generalized pseudospectral method. The procedure is applied to a case study of the resonance-enhanced MPI and HHG of atomic hydrogen driven by an intense frequency-comb laser field. Our study shows that both the MPI and HHG rates can be coherently controlled by tuning the laser parameters such as the pulse-to-pulse carrier-envelope-phase (CEP) shift. In particular, both the MPI and HHG rates exhibit dramatic enhancement by tuning the CEP shift, due to the phenomenon of multiphoton resonances.
\end{abstract}

DOI: 10.1103/PhysRevA.87.043407

PACS number(s): 32.80.Qk, 42.65.Ky, 31.15.A-, 42.62.Eh

\section{INTRODUCTION}

In the past decade, advancements in femtosecond laserbased optical frequency combs have had important impacts in high-precision optical frequency spectroscopy [1-5], the development of an optical atomic clock [6-11], the determination of fundamental constants [12,13], the testing of quantum electrodynamics [14-17], and highly sensitive molecular detection $[18,19]$. Due to its ability to precisely and directly link optical and microwave frequencies [20,21], the optical frequency comb provides a "ruler" in the frequency domain with which an unknown optical frequency can be measured. The femtosecond frequency-comb techniques also play a remarkable role in ultrafast science [22,23]. Control of the phase of few-cycle pulses [24-27] provides a powerful tool for the coherent control of atomic and molecular dynamics [28-30], and the generation of soft-X-ray attosecond pulses by high-order-harmonic generation (HHG) [31-33], which depend strongly on the carrier-envelope phase (CEP). At present, extending the frequency combs into the extreme ultraviolet (wavelength below $100 \mathrm{~nm}$ ) spectral region is highly desirable due to the lack of powerful continuous-wave lasers in that spectral region.

Recently, coherent extreme ultraviolet (XUV) [34] and vacuum ultraviolet (VUV) [35] radiation at a repetition frequency of more than $100 \mathrm{MHz}$, a 1000-fold improvement over previous experiments, has been generated via HHG. The behavior of the frequency-comb structure and the coherence in $\mathrm{HHG}$ in the VUV-XUV regimes were first explored theoretically by Carrera et al. by means of the accurate solution of the time-dependent Schrödinger equation (TDSE) $[36,37]$ and the time-frequency spectrum. It was found that a nested comb structure appears within each of the harmonics,

\footnotetext{
*sichu@ku.edu
}

ranging from the first harmonic all the way to the cutoff harmonic [36,37]. More recently, it was shown by several experiments $[15,38-40]$ that the frequency-comb structure and coherence can indeed survive in very high-order harmonics and in the situation in which substantial ionization occurs in the presence of high-intensity laser fields. In these experiments, the femtosecond enhancement cavity technique (for a recent review, see Ref. [41]) is used to achieve significantly higher repetition rates with suitable pulse energies. For example, Cingöz et al. [39] reported the generation of XUV frequency combs up to 27 th harmonic order (wavelengths of $40 \mathrm{~nm}$ ) by coupling a high-power near-infrared frequency comb to a robust femtosecond enhancement cavity. Alternatively, Kandula et al. $[15,40]$ demonstrated the frequency comb structure and phase coherence in 15th harmonics (wavelengths of $51 \mathrm{~nm}$ ) by employing Ramsey spectroscopy [42,43]. In addition, Son and Chu [44] have recently presented a theoretical extension of the many-mode Floquet theory (MMFT) [45-49] for the nonperturbative treatment of the coherent control of multiphoton resonance dynamics and the enhancement of HHG driven by intense frequency-comb laser fields. Their study of a simple two-level system shows that HHG driven by an intense frequency-comb laser has the comb structure with the same repetition frequency and different offset for each harmonic, and it can be dramatically enhanced by tuning the CEP shift due to multiphoton resonance among all the comb frequencies. This multiphoton-resonant enhancement approach promises an alternative method to achieve a powerful frequency comb structure in the VUV and/or XUV region without increasing the intensity of the driving frequency-comb laser fields.

However, the two-level model does not take into account the effects of multilevel structure and ionization, which are inherent in real atomic and/or molecular systems driven by intense laser fields. These effects may distract the multiphoton resonant enhancement of the HHG driven by intense 
frequency-comb laser fields. As far as we know, it is still an open question whether the HHG in real atomic and/or molecular systems can be efficiently enhanced when they are driven by intense frequency-comb laser fields. In this paper, we present a fully $a b$ initio investigation of the coherent control and enhancement of HHG and multiphoton ionization (MPI) driven by intense frequency-comb laser fields in real atomic and/or molecular systems. In the limit of a long train of short pulses or an infinite number of pulses, we can extend the MMFT for the accurate treatment of the interaction of the frequency-comb laser fields with atomic and/or molecular systems. The MMFT allows the exact transformation of the timedependent polychromatic or quasiperiodic Hamiltonian into an equivalent time-independent generalized Floquet Hamiltonian $H_{F}$. Then, we apply the complex-scaling (CS) transformation to the generalized Floquet Hamiltonian, converting $H_{F}$ into a non-Hermitian Floquet Hamiltonian, allowing the inclusion of both bound and continuum states and the calculation of the complex quasienergies and quasienergy eigenfunctions $[45,50]$. The real part of the quasienergy provides the ac-Stark shifted level energy, while the imaginary part gives rise to the total MPI rate $[45,50]$. Finally, the generalized pseudospectral (GPS) method [45,51] is extended for the optimal spatial discretization of $H_{F}$, allowing accurate and efficient calculation of the HHG and MPI rates. In this paper, we extend our non-Hermitian MMFT to the accurate nonperturbative treatment of the coherent control and enhancement of HHG and MPI of atomic hydrogen in the presense of intense frequency-comb laser fields.

The paper is organized as follows: In Sec. II, we present the non-Hermitian MMFT for the treatment of the interaction of frequency-comb laser fields with real atomic and/or molecular systems. In Sec. III, we apply our approach to the study of the coherent control and enhancement of HHG and MPI rates of atomic hydrogen driven by intense frequency-comb laser fields. This is followed by a conclusion in Sec. IV.

\section{NON-HERMITIAN MMFT APPROACH FOR THE NONPERTURBATIVE TREATMENT OF MULTIPHOTON PROCESSES OF ATOMIC AND MOLECULAR SYSTEMS DRIVEN BY FREQUENCY-COMB LASER FIELDS}

The frequency-comb laser is generated by a train of equalspacing short laser pulses. Consider a temporal train of short laser pulses with the carrier (fundamental) angular frequency $\omega_{c}$ and the time interval $\tau$ between pulses. The frequencycomb laser consists of spectral comb lines in the frequency domain [21],

$$
\omega_{m}=m \omega_{r}+\omega_{\delta},
$$

where $\omega_{r}(=2 \pi / \tau)$ is the repetition angular frequency, $m$ is an integer index, and $\omega_{\delta}$ is the offset angular frequency $(0 \leqslant$ $\left.\omega_{\delta} \leqslant \omega_{r}\right)$. Without loss of generality, these comb frequencies can be written as

$$
\omega_{k}=\omega_{0}+k \omega_{r}
$$

where $k$ is an integer index and $\omega_{0}$ is the main angular frequency defined to be the frequency in $\omega_{m}$ whose frequency amplitude is the maximum in the frequency domain. The relation between $\omega_{0}$ and $\omega_{c}$ is given by

$$
\omega_{0}=\left[\frac{\omega_{c}-\omega_{\delta}}{\omega_{r}}\right] \omega_{r}+\omega_{\delta},
$$

where [ ] is the round function. In general, the carrier frequency $\omega_{c}$ is not necessarily one of the comb frequencies nor does it equal $\omega_{0}$. Due to the incommensuration between the time period $\left(=2 \pi / \omega_{c}\right)$ of the carrier wave and the time interval $\tau$ of the pulse envelope, there is a carrier-envelope phase (CEP) shift $\Delta \phi$ from pulse to pulse. The CEP shift $\Delta \phi$ plays a key role in stabilizing the comb structure in the current modelocking laser technique [25], and it determines all absolute positions of frequencies in the comb structure by setting the offset frequency $\omega_{\delta}=\Delta \phi / \tau$.

The electric field for a train of pulses with the CEP shift in the time domain is expressed by

$$
E(t)=\sum_{n} f(t-n \tau) e^{i\left(\omega_{c} t-n \omega_{c} \tau+n \Delta \phi\right)},
$$

where $f(t)$ is the envelope function for one pulse in the time domain. In the ideal case in which an infinite number of pulses are added up, Eq. (4) reduces to the summation of exponentials of discrete comb frequencies with their individual field amplitudes,

$$
E(t)=\sum_{k=-\infty}^{\infty} E_{k} e^{i \omega_{k} t},
$$

where $E_{k}$ is the field amplitude corresponding to the frequency comb with $\omega_{k}$. Let the envelope function have the Gaussian form $f(t)=f_{0} e^{-t^{2} / 2 \sigma^{2}}$, where $f_{0}$ is the peak field amplitude and $\sigma$ is the standard deviation of a Gaussian function. Then the field amplitude $E_{k}$ corresponding to $\omega_{k}$ is [44]

$$
E_{k}=\frac{f_{0} \sigma \omega_{r}}{\sqrt{2 \pi}} e^{-\sigma^{2}\left(\omega_{0}-\omega_{c}+k \omega_{r}\right)^{2} / 2} .
$$

The number of frequency combs in Eq. (5) determines the dimension of the Floquet Hamiltonian, which is required to be a finite number for diagonalization in numerical calculations. Thus a finite integer number $N$ is chosen such that

$$
E(t) \simeq \sum_{k=-N}^{N} E_{k} e^{i \omega_{k} t},
$$

to reproduce approximately well the total field and make sure the calculation is accessible and convergent. In our calculation, $N$ is chosen to ignore combs with amplitudes less than $1 \times 10^{-15}$ a.u. (corresponding to an intensity of $3.51 \times 10^{-14} \mathrm{~W} / \mathrm{cm}^{2}$ ). For instance, we take $N=14$ when using a train of Gaussian 20 fs FWHM pulses with peak intensity $1 \times 10^{12} \mathrm{~W} / \mathrm{cm}^{2}$ and repetition frequency $10 \mathrm{THz}$.

To investigate the interaction of an atomic or molecular system with the frequency-comb laser generated by a finite number of pulses, Eq. (4) has been employed to solve the time-dependent Schrödinger equation in the time domain [36,37]. On the other hand, for the ideal comb laser generated by an infinite number of pulses, it is more expedient to avoid the time propagation of the wave function. The many-mode Floquet theory (MMFT) [44-49] for the polychromatic or quasiperiodic time-dependent Schrödinger 
equation provides the exact solution in terms of the generalized many-mode quasienergy eigenvalues and eigenfunctions, without the need to perform the time-consuming propagation of the wave function. Consider now the interaction of an atomic or molecular system with a linearly polarized frequency-comb laser field in the $z$ direction. The total Hamiltonian including $(2 N+1)$ comb frequencies is given by

$$
\begin{aligned}
H(\boldsymbol{r}, t) & =\hat{H}_{0}(\boldsymbol{r})-\sum_{k=-N}^{N} \mu(\boldsymbol{r}) \cdot \boldsymbol{E}_{k} \cos \omega_{k} t \\
& =\hat{H}_{0}(\boldsymbol{r})-\sum_{k=-N}^{N} \frac{1}{2} \hat{z} E_{k}\left[e^{i\left(\omega_{0}+k \omega_{r}\right) t}+e^{-i\left(\omega_{0}+k \omega_{r}\right) t}\right]
\end{aligned}
$$

where $\hat{H}_{0}(\boldsymbol{r})$ is the unperturbed Hamiltonian of the atomic or molecular system and $\mu(\boldsymbol{r})$ is the electric dipole moment operator. Note that the time-dependent Hamiltonian in Eq. (8) is bichromatic, containing two independent frequencies $\omega_{0}$ and $\omega_{r}$.

By employing the MMFT and the complex-scaling transformation, the bichromatic Hamiltonian in Eq. (8) can be converted into an equivalent time-independent non-Hermitian generalized Floquet Hamiltonian $H_{F}$. Discretizing $H_{F}$ by means of the GPS method, and solving the corresponding complex eigenvalue problem, we can obtain a set of complex quasienergies and the corresponding left and right eigenvectors which possess the property of biorthogonality [45]. The eigenvalues and eigenvectors can be enumerated with four indexes, the first one corresponding to the radial eigenstate $(i)$, the second one to the angular momentum $(l)$, and the other two to Fourier components of $n$ and $m$ for $\omega_{0}$ and $\omega_{r}$, respectively,

$$
|\alpha n m\rangle=|\alpha\rangle \otimes|n\rangle \otimes|m\rangle
$$

where $|\alpha\rangle=|i l\rangle$ is the state index.

The generalized Floquet matrix has the following explicit form:

$$
\sum_{\beta} \sum_{n^{\prime}} \sum_{m^{\prime}}\left\langle\alpha n m\left|H_{F}\right| \beta n^{\prime} m^{\prime}\right\rangle\left\langle\beta n^{\prime} m^{\prime} \mid \lambda\right\rangle=\lambda\langle\alpha n m \mid \lambda\rangle,
$$

where $\lambda$ is the quasienergy eigenvalue and $|\lambda\rangle$ is the corresponding eigenvector. In general, the quasienergy $\lambda$ is complex-valued,

$$
\lambda=\mathcal{E}-i \frac{\Gamma}{2},
$$

where $\mathcal{E}$ (the real part of the quasienergy) describes the position of the ac-Stark shifted energy level in the external field, and $\Gamma$ is the total MPI rate. The two-mode Floquet matrix $H_{F}$ is constructed by

$$
\begin{aligned}
& \left\langle i l n m\left|H_{F}\right| j l^{\prime} n^{\prime} m^{\prime}\right\rangle \\
& \quad=H_{i l, j l^{\prime}}^{\left[n-n^{\prime}, m-m^{\prime}\right]}+\left(n \omega_{0}+m \omega_{r}\right) \delta_{i, j} \delta_{l, l^{\prime}} \delta_{n, n^{\prime}} \delta_{m, m^{\prime}},
\end{aligned}
$$

where

$$
\begin{gathered}
H_{i l, j l^{\prime}}^{\left[n-n^{\prime}, m-m^{\prime}\right]}=\left[H_{i, j}^{0}\right]_{l} \delta_{l, l^{\prime}} \delta_{n, n^{\prime}} \delta_{m, m^{\prime}}+\sum_{k=-N}^{N}\left[V_{i, j}^{(k)}\right]_{l, l^{\prime}}\left(\delta_{n+1, n^{\prime}} \delta_{m+k, m^{\prime}}+\delta_{n-1, n^{\prime}} \delta_{m-k, m^{\prime}}\right), \\
{\left[V_{i, j}^{(k)}\right]_{l, l^{\prime}}=-\frac{1}{2} E_{k} r_{i} \frac{l}{\sqrt{(2 l+1)\left(2 l^{\prime}+1\right)}} \delta_{i, j} \delta_{l-1, l^{\prime}}-\frac{1}{2} E_{k} r_{i} \frac{l^{\prime}}{\sqrt{(2 l+1)\left(2 l^{\prime}+1\right)}} \delta_{i, j} \delta_{l+1, l^{\prime}},}
\end{gathered}
$$

and $\left[H_{i, j}^{0}\right]_{l}$ is the radial Hamiltonian matrix for the angular momentum $l$.

The $H_{F}$ matrix has a block band-diagonal structure with infinite-dimensional diagonal blocks and subdiagonal blocks corresponding to the quasienergy states of an atomic and/or molecular system and interactions between the system and the $(2 N+1)$ comb frequencies, respectively. The structure of $H_{F}$ for the case of a linearly polarized field is as follows:

$$
H_{F}=\left(\begin{array}{ccccccc}
\ddots & \multicolumn{7}{c}{} & & & & \vdots & \\
& A+2 \omega_{0} I & B & 0 & 0 & 0 & \cdots \\
& B^{T} & A+\omega_{0} I & B & 0 & 0 & \\
& 0 & B^{T} & A & B & 0 & \\
\cdots & 0 & 0 & B^{T} & A-\omega_{0} I & B & \\
& 0 & 0 & 0 & B^{T} & A-2 \omega_{0} I & \\
\vdots & & & & & \ddots
\end{array}\right),
$$

where

$$
A=\left(\begin{array}{ccccccc}
\ddots & & & & & \vdots & \\
& Z+2 \omega_{r} I & 0 & 0 & 0 & 0 & \ldots \\
0 & Z+\omega_{r} I & 0 & 0 & 0 & \\
& 0 & 0 & Z & 0 & 0 & \\
\cdots & 0 & 0 & 0 & Z-\omega_{r} I & 0 & \\
& 0 & 0 & 0 & 0 & Z-2 \omega_{r} I & \\
\vdots & & & & & \ddots
\end{array}\right)
$$




$$
B=\left(\begin{array}{ccccccc}
\ddots & & & & & \vdots & \\
& Y_{0} & Y_{1} & Y_{2} & Y_{3} & Y_{4} & \cdots \\
& Y_{-1} & Y_{0} & Y_{1} & Y_{2} & Y_{3} & \\
& Y_{-2} & Y_{-1} & Y_{0} & Y_{1} & Y_{2} & \\
& Y_{-3} & Y_{-2} & Y_{-1} & Y_{0} & Y_{1} & \\
\cdots & Y_{-4} & Y_{-3} & Y_{-2} & Y_{-1} & Y_{0} & \\
& \vdots & & & & & \ddots
\end{array}\right) .
$$

The matrices of $Y_{k}$ and $Z$ which form blocks in $A$ and $B$ have the following forms:

$$
\begin{gathered}
Y_{k}=\left(\begin{array}{cccc}
0 & {\left[V_{i, j}^{k}\right]_{S, P}} & 0 & \cdots \\
{\left[V_{i, j}^{k}\right]_{P, S}} & 0 & {\left[V_{i, j}^{k}\right]_{P, D}} & \\
0 & {\left[V_{i, j}^{k}\right]_{D, P}} & 0 & \\
\vdots & & & \ddots
\end{array}\right), \\
Z=\left(\begin{array}{cccc}
{\left[H_{i, j}^{0}\right]_{S}} & 0 & 0 & \cdots \\
0 & {\left[H_{i, j}^{0}\right]_{P}} & 0 & \\
0 & 0 & {\left[H_{i, j}^{0}\right]_{D}} & \\
\vdots & & & \ddots
\end{array}\right) .
\end{gathered}
$$

After solving the eigenvalue problem of the generalized non-Hermitian Floquet matrix, the time-averaged transition probability can be computed from the quasienergy eigenvectors [46],

$$
\bar{P}_{\alpha \rightarrow \beta}=\sum_{n, m} \sum_{\gamma, n^{\prime}, m^{\prime}}\left|\left\langle\beta n m \mid \lambda_{\gamma n^{\prime} m^{\prime}}\right\rangle\left\langle\lambda_{\gamma n^{\prime} m^{\prime}} \mid \alpha 00\right\rangle\right|^{2},
$$

which has the maximum values at avoided crossings of quasienergies associated with multiphoton resonance transitions [47]. The induced dipole moment can be expanded in double Fourier series,

$$
\boldsymbol{d}(t)=\sum_{n, m} \boldsymbol{d}_{n, m} e^{-i\left(n \omega_{0}+m \omega_{r}\right) t} .
$$

Given the value of $n$ and $m$, the angular frequency $\omega$ is determined by $\omega=n \omega_{0}+m \omega_{r}$. The harmonic generation spectra in the length form can be expressed by

$$
\begin{aligned}
P\left(n \omega_{0}+m \omega_{r}\right) & =\frac{4}{6 \pi c^{3}}\left|\boldsymbol{d}_{n, m}\right|^{2} \\
& =\frac{4}{6 \pi c^{3}}\left|\sum_{\alpha, \beta} \sum_{n^{\prime}, m^{\prime}}\left\langle\lambda_{\alpha, n^{\prime}-n, m^{\prime}-m}|\hat{z}| \lambda_{\beta, n^{\prime}, m^{\prime}}\right\rangle\right|^{2} .
\end{aligned}
$$

Here the harmonic order is defined by $\omega / \omega_{c}$ and can be a fractional number because of the comb structure of frequencies.

\section{RESULTS AND DISCUSSIONS}

In this section, we investigate the HHG and MPI of atomic hydrogen driven by frequency-comb laser fields by employing the complex-scaling non-Hermitian MMFT. First, we focus on the $1 s$ and $2 p$ states of atomic hydrogen as a case study.
The laser parameters used are peak intensity $1 \times 10^{12} \mathrm{~W} / \mathrm{cm}^{2}$ and repetition frequency $10 \mathrm{THz}$ (corresponding to $\omega_{r}=$ $1.51983 \times 10^{-3}$ a.u. and pulse separation $\tau=0.1 \mathrm{ps}$ ) generated from a train of Gaussian pulses with $20 \mathrm{fs}$ full width at half maximum (FWHM). Figure 1(a) shows the quasienergies around -0.5 a.u., and Fig. 1(b) shows the MPI rates as a function of the carrier frequency varying from 0.12 to 0.13 a.u. (corresponding to a carrier wavelength from 380 to $350 \mathrm{~nm}$, which is around three-photon dominated resonance between $1 s$ and $2 p$ states). The main frequency $\omega_{0}$ is set to be the carrier frequency $\omega_{c}$, so that the offset angular frequency $\omega_{\delta}$ is chosen to be a reminder of $\omega_{c}$ divided by $\omega_{r}$. The quasiperiodic structure of the quasienergies can be represented by $[46,47]$

$$
\mathcal{E}_{\gamma n m}=\mathcal{E}_{\gamma}+n \omega_{0}+m \omega_{r},
$$

where $n$ is an even integer when $\gamma=1 s$ and an odd integer when $\gamma=2 p$ due to parity consideration. The corresponding time-averaged transition probabilities from the $1 \mathrm{~s}$ state to the $2 p$ state are shown in Fig. 1(c). A cluster of resonances [Fig. 1(c)] and MPI enhancement [Fig. 1(b)] are observed in

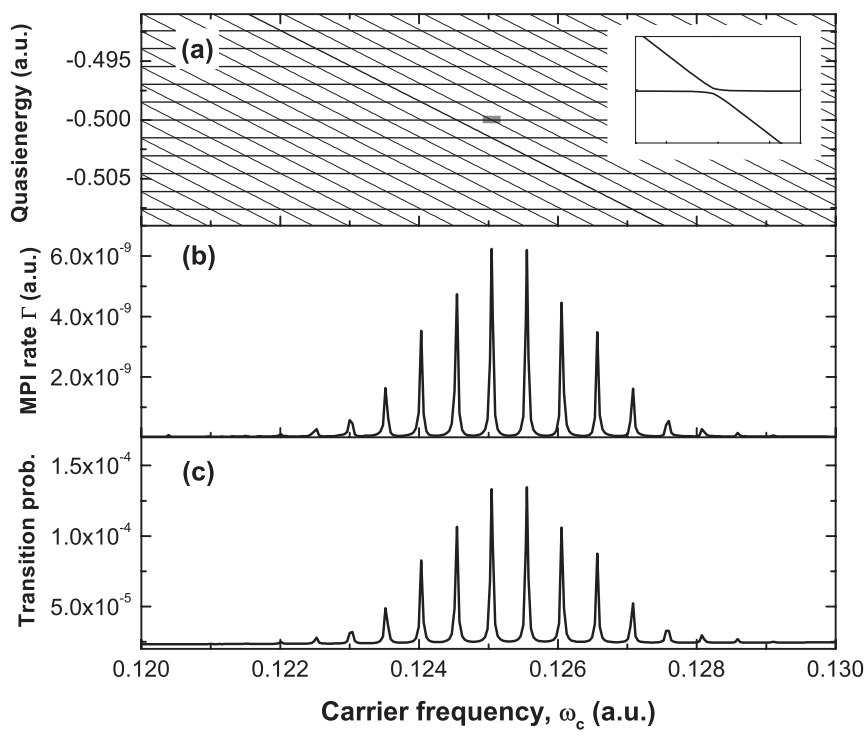

FIG. 1. (a) Quasienergies and (b) MPI rates as a function of carrier frequency, for atomic hydrogen driven by frequency-comb laser fields with peak intensity $1 \times 10^{12} \mathrm{~W} / \mathrm{cm}^{2}$ and repetition frequency 10 $\mathrm{THz}$ of $20 \mathrm{fs}$ FWHM Gaussian pulses. The enlarged quasienergy plot shown in the inset of (a) corresponds to the gray box in (a). (c) The corresponding time-averaged transition probabilities from the $1 s$ state to the $2 p$ state. 


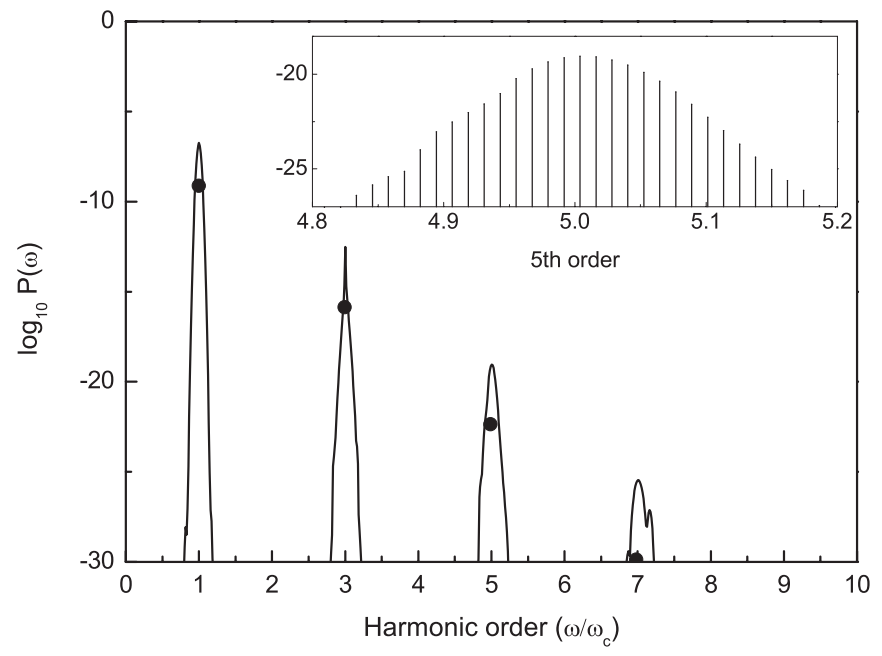

FIG. 2. HHG power spectrum of atomic hydrogen driven by the frequency-comb laser with CEP shift $\Delta \phi / 2 \pi=0.276364$. Each harmonic has a nested comb structure as shown in the inset for the fifth harmonic. For clarity, all comb peaks are connected by a line. The laser parameters used are peak intensity $1 \times 10^{12} \mathrm{~W} / \mathrm{cm}^{2}$, carrier wavelength $364.66 \mathrm{~nm}$, and repetition frequency $10 \mathrm{THz}$ of $20 \mathrm{fs}$ FWHM Gaussian pulses. The HHG spectra driven by a one-mode laser with the same carrier wavelength $(364.66 \mathrm{~nm})$ and the corresponding root-mean-square field strength are represented by dots.

the vicinity of resonance positions due to the comb structure of frequencies. The positions of resonances and enhancement are at the avoided crossings of quasienergies shown in Fig. 1(a). For instance, the enlarged avoided crossing labeled by the gray box in Fig. 1(a) is shown in the inset in Fig. 1(a). The coherent control and enhancement of HHG will be discussed, followed by the study of the coherent control and enhancement of the MPI rate.

Figure 2 presents the HHG power spectrum of atomic hydrogen driven by a frequency-comb laser. The carrier wavelength is fixed at $364.66 \mathrm{~nm}\left(\omega_{c}=0.1249495\right.$ a.u. $)$ and the CEP shift is fixed at $\Delta \phi / 2 \pi=0.276364$ for nearresonance. Other laser parameters are the same as those in Fig. 1. For comparison, HHG driven by a one-mode laser with the same carrier wavelength $(364.66 \mathrm{~nm})$ and the corresponding root-mean-square field strength is computed by one-mode Floquet calculation, and plotted with dots in Fig. 2. As predicted by Ref. [44], the HHG driven by a frequency-comb laser is orders of magnitude higher than that driven by a one-mode laser. A nested comb structure appears within each of the harmonics, which arises from quantum interferences among induced dipoles generated by the incident sequence of laser pulses [36]. For instance, the comb structure within the fifth-order harmonic is shown in the inset in Fig. 2. Each of the harmonic orders has the same repetition angular frequency $\omega_{r}$ and the offset angular frequency $n \omega_{\delta}$ for the $n$ th-order harmonic.

The enhancement of HHG and the comb structure in harmonics can be explained by multiphoton resonances that occur due to the multitude of frequencies at the same time. For a general consideration, we discuss the $n$-photon resonance conditions of monochromatic and frequency-comb fields. For the atomic hydrogen driven by the monochromatic field, the $n$-photon resonance condition for $1 s-2 p$ excitation is

$$
\omega_{\mathrm{res}}=n \omega,
$$

where $\omega_{\text {res }}$ is the $1 s-2 p$ resonance frequency of atomic hydrogen in the presence of a monochromatic field. The resonance frequency is hard to reach since the ac-Stark shifts of levels depend not only on the intensity, but also on the carrier frequency of the monochromatic field. On the other hand, the comb frequencies in the frequency-comb laser can be expressed as

$$
\omega=m \omega_{r}+\omega_{\delta},
$$

where the offset $\omega_{\delta}$ is controlled by the CEP shift $\Delta \phi$. And the frequency comb spectrum for the $n$ th-order harmonic can be expressed as

$$
\omega_{k}^{\prime}=n \omega_{\delta}+k \omega_{r}
$$

where $k$ is an integer index. As the CEP shift is varied, the resonance position is retained since the pulse energy is conserved. The retained resonance position makes it much easier than tuning the carrier frequency in a monochromatic field to reach the resonance condition. The $n$-photon resonance condition for comb frequencies with the offset $\omega_{\delta}$ is expressed as

$$
\omega_{\text {res }} \equiv n\left(m \omega_{r}+\omega_{\delta}\right) \quad\left(\bmod \omega_{r}\right)
$$

which means the reminders of $\omega_{\text {res }}$ and $n\left(m \omega_{r}+\omega_{\delta}\right)$ are the same when divided by $\omega_{r}$. And the CEP shift for $n$-photon resonance is given by

$$
\Delta \phi_{j}=2 \pi\left(\frac{\omega_{\delta}}{\omega_{r}}+\frac{j}{n}\right) \quad(0 \leqslant j<n) .
$$

When one comb frequency fulfills the multiphoton resonance condition, many combinations of comb frequencies can simultaneously contribute to that multiphoton resonance, leading to the dramatic enhancement of the HHG spectrum.

Table I lists the HHG power spectrum values of the maximum peak with several peak intensities of the frequencycomb lasers. Other laser parameters are the same as those in Fig. 2. The near- and off-resonance conditions are controlled by varying the CEP shift. It shows that harmonic peaks can be dramatically enhanced when the three-photon resonance condition is fulfilled. For the case of $I=1 \times 10^{11} \mathrm{~W} / \mathrm{cm}^{2}$, the HHG power spectra are enhanced by about $10^{4}$ times, while for the case of $I=1 \times 10^{12}$ and $3 \times 10^{12} \mathrm{~W} / \mathrm{cm}^{2}$, the HHG power spectra are enhanced by about $10^{2}$ and 10 times, respectively. Here we demonstrate that the HHG spectra in atomic hydrogen can be efficiently enhanced when driven by intense frequency-comb laser fields. However, the enhancement factors in atomic hydrogen are considerably smaller than those in the two-level system presented by Son and Chu [44]. The major cause is that the excitation from ground level to multilevels, which is not included in the two-level system, makes the frequency dependence of the HHG process in atomic hydrogen on the $1 s-2 p$ resonance condition not as strong as that in the two-level systems.

We also present the MPI rate as a function of the CEP shift $\Delta \phi$ with peak intensities $3 \times 10^{12}$ and $1 \times 10^{12} \mathrm{~W} / \mathrm{cm}^{2}$ in Figs. 3(a) and 3(c), respectively. The MPI rate reaches the maximum values when the CEP shift $\Delta \phi=(0.337391+n / 3) \times$ $2 \pi$ with the peak intensity $3 \times 10^{12} \mathrm{~W} / \mathrm{cm}^{2}$, and it reaches 
TABLE I. Effects on the HHG power spectra by varying the CEP shift $\Delta \phi . n$ is the harmonic order of the maximum peak for each harmonic and $P\left(n \omega_{c}\right)$ is the corresponding HHG power spectrum values at $\omega=n \omega_{c}$. The label A indicates the near-resonance cases: $\Delta \phi / 2 \pi=0.249067$, 0.276364 , and 0.337391 corresponding to the laser peak intensities $1 \times 10^{11}, 1 \times 10^{12}$, and $3 \times 10^{12} \mathrm{~W} / \mathrm{cm}^{2}$, respectively, while B indicates the off-resonance cases with $\Delta \phi / 2 \pi=0.1$. The numbers in brackets indicate the power of 10 . The other laser parameters used are the same as those in Fig. 2.

\begin{tabular}{|c|c|c|c|c|c|c|c|c|c|c|c|}
\hline \multicolumn{4}{|c|}{$1 \times 10^{11} \mathrm{~W} / \mathrm{cm}^{2}$} & \multicolumn{4}{|c|}{$1 \times 10^{12} \mathrm{~W} / \mathrm{cm}^{2}$} & \multicolumn{4}{|c|}{$3 \times 10^{12} \mathrm{~W} / \mathrm{cm}^{2}$} \\
\hline \multicolumn{2}{|r|}{ A } & \multicolumn{2}{|r|}{ B } & \multicolumn{2}{|r|}{ A } & \multicolumn{2}{|r|}{ B } & \multicolumn{2}{|r|}{ A } & \multicolumn{2}{|r|}{ B } \\
\hline$n$ & $P\left(n \omega_{c}\right)$ & $n$ & $P\left(n \omega_{c}\right)$ & $n$ & $P\left(n \omega_{c}\right)$ & $n$ & $P\left(n \omega_{c}\right)$ & $n$ & $P\left(n \omega_{c}\right)$ & $n$ & $P\left(n \omega_{c}\right)$ \\
\hline 3.001 & $3.17[-14]$ & 2.996 & $2.23[-18]$ & 3.002 & $3.04[-13]$ & 2.996 & $1.93[-15]$ & 3.005 & $6.80[-13]$ & 2.996 & $4.88[-14]$ \\
\hline 5.002 & $9.00[-23]$ & 4.969 & $5.32[-27]$ & 5.004 & $8.87[-20]$ & 4.969 & $3.85[-22]$ & 5.020 & $2.12[-18]$ & 5.030 & $8.07[-20]$ \\
\hline 7.003 & $3.59[-31]$ & 6.954 & $2.77[-35]$ & 7.005 & $3.53[-26]$ & 6.954 & $1.98[-28]$ & 7.023 & $7.78[-24]$ & 6.942 & $5.46[-25]$ \\
\hline
\end{tabular}

the maximum values when the CEP shift $\Delta \phi=(0.249067+$ $n / 3) \times 2 \pi$ with the peak intensity $1 \times 10^{12} \mathrm{~W} / \mathrm{cm}^{2}$. The MPI rates driven by a one-mode laser with the same carrier wavelength $(364.66 \mathrm{~nm})$ and the corresponding root-mean-square field strength are computed by one-mode Floquet calculation. The MPI rates driven by a one-mode laser, $1.0487 \times 10^{-9}$ and $3.1435 \times 10^{-11}$ a.u. corresponding to Figs. 3(a) and 3(c), respectively, are much smaller than those driven by frequency-comb laser fields. From Figs. 3(a) and 3(c), one can see that the MPI rate reaches the maximum value three times by varying the CEP shift, and these three peak positions are separated by $2 \pi / 3$ exactly, according to Eq. (28). The MPI rate can be considered as consisting of two main contributions: one is the direct ionization from the $1 s$ state to continuum and the other is ionization from the "intermediate" $2 p$ state that is populated via multiphoton excitation. Since it is much easier to ionize the electron from the $2 p$ state than directly from the $1 s$ state, the MPI rate can be dramatically increased when the condition of the resonance excitation of the $2 p$ state is fulfilled. For comparison, we present the maximum peak value within the fifth harmonic as a function of the CEP shift $\Delta \phi$ with peak intensities $3 \times 10^{12} \mathrm{~W} / \mathrm{cm}^{2}$ [Fig. 3(b)] and $1 \times 10^{12} \mathrm{~W} / \mathrm{cm}^{2}$ [Fig. 3(b)], respectively. The dependence of the MPI rate, Fig. 3(a) [or Fig. 3(c)], and the maximum HHG peak value, Fig. 3(b) [or Fig. 3(d)], on the CEP shift are in perfect agreement, indicating that both the HHG and MPI rate can be dramatically enhanced via the multiphoton resonance excitation of excited states achieved by varying the CEP shift. Here we note that the enhancement of the HHG and MPI rates is achieved without increasing the intensity of the driving frequency-comb laser fields.

To demonstrate the generality of the multiphoton resonance enhancement, we also investigate several cases for carrier wavelengthes corresponding to different multiphotonresonance conditions. The other carrier wavelengths used are $307.65 \mathrm{~nm}$ (corresponding to three-photon dominant resonance between $1 s$ and $3 p$ states), $670.76 \mathrm{~nm}$ (five-photon dominant resonance between $1 s$ and $2 p$ states), and $850.54 \mathrm{~nm}$
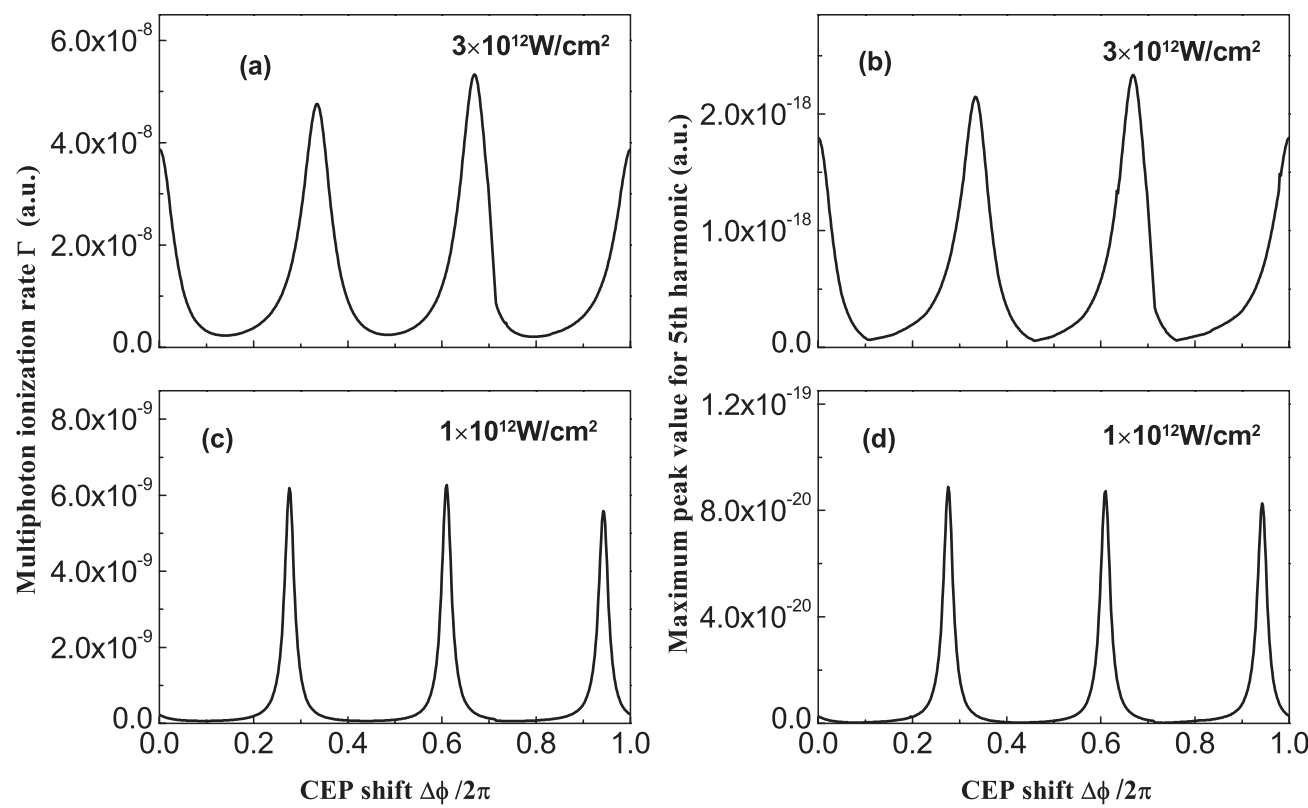

FIG. 3. (a) Multiphton ionization rate and (b) maximum peak value within the fifth harmonic as a function of the CEP shift $\Delta \phi$ with peak intensity $3 \times 10^{12} \mathrm{~W} / \mathrm{cm}^{2}$. (c) Multiphoton ionization rate and (d) maximum peak value within the fifth harmonic as a function of the CEP shift $\Delta \phi$ with peak intensity $1 \times 10^{12} \mathrm{~W} / \mathrm{cm}^{2}$. The other laser parameters are the same as those in Fig. 2. 
TABLE II. The HHG power spectra of different carrier wavelengths by tuning the CEP shift $\Delta \phi . n$ is the harmonic order of the maximum peak for each harmonic and $P\left(n \omega_{c}\right)$ is the corresponding HHG power spectrum values at $\omega=n \omega_{c}$. The label A indicates the near-resonance cases: $\Delta \phi / 2 \pi=0.156574,0.058317$, and 0.084002 corresponding to the laser carrier wavelengths $307.65,670.76$, and $850.54 \mathrm{~nm}$, respectively, while $\mathrm{B}$ indicates the off-resonance cases with $\Delta \phi / 2 \pi=0.1$. The numbers in brackets indicate the power of 10. The other laser parameters used are the same as those in Fig. 2.

\begin{tabular}{|c|c|c|c|c|c|c|c|c|c|c|c|}
\hline \multicolumn{4}{|c|}{$307.65 \mathrm{~nm}$} & \multicolumn{4}{|c|}{$670.76 \mathrm{~nm}$} & \multicolumn{4}{|c|}{$850.54 \mathrm{~nm}$} \\
\hline \multicolumn{2}{|r|}{ A } & \multicolumn{2}{|r|}{ B } & \multicolumn{2}{|r|}{$\mathrm{A}$} & \multicolumn{2}{|r|}{ B } & \multicolumn{2}{|r|}{ A } & \multicolumn{2}{|r|}{ B } \\
\hline$n$ & $P\left(n \omega_{c}\right)$ & $n$ & $P\left(n \omega_{c}\right)$ & $n$ & $P\left(n \omega_{c}\right)$ & $n$ & $P\left(n \omega_{c}\right)$ & $n$ & $P\left(n \omega_{c}\right)$ & $n$ & $P\left(n \omega_{c}\right)$ \\
\hline 3.001 & $2.06[-12]$ & 3.000 & $1.73[-15]$ & & & & & & & & \\
\hline 4.994 & $2.85[-19]$ & 4.982 & $2.82[-22]$ & 5.013 & $2.79[-17]$ & 4.997 & $1.56[-20]$ & & & & \\
\hline \multirow[t]{3}{*}{6.989} & $4.67[-26]$ & 6.975 & $4.50[-29]$ & 7.023 & $8.02[-22]$ & 6.875 & $3.59[-24]$ & 6.996 & $4.61[-22]$ & 6.999 & $1.12[-25]$ \\
\hline & & & & 9.012 & $6.48[-26]$ & 8.938 & $6.46[-28]$ & 8.959 & $2.14[-23]$ & 8.962 & $1.14[-26]$ \\
\hline & & & & & & & & 10.779 & $2.88[-27]$ & 10.954 & $1.72[-30]$ \\
\hline
\end{tabular}

(seven-photon dominant resonance between $1 s$ and $2 p$ states), and other laser parameters are the same as those in Fig. 2. The HHG power spectrum values of the maximum peak for the near- and off-resonance cases with these carrier wavelengthes are listed in Table II. For the case of three-photon dominant resonance between $1 s$ and $3 p$ states, the enhancement factor for $n \geqslant 3$ is about $10^{3}$ times, while for the case of five- and seven-photon dominant resonance between $1 s$ and $2 p$ states, the enhancement factors are about $10^{2}$ times for $n \geqslant 5$ and $10^{3}$ times for $n \geqslant 7$, respectively.

\section{CONCLUSION}

In this paper, we have presented a fully ab initio theoretical investigation of HHG and MPI from real atomic and/or molecular systems, for instance atomic hydrogen, driven by an intense frequency-comb laser field. The bound and continuum states of the atomic and/or molecular systems are treated accurately and efficiently by applying the complex-scaling generalized pseudospectral method, and their interaction with frequency-comb laser fields is accurately solved by employing the many-mode Floquet theory. It is shown that the HHG and MPI rates can be dramatically enhanced by tuning the pulse-to-pulse CEP shift without increasing the intensity of the driving laser field. Moreover, it is demonstrated that the enhancement is due to the phenomenon of multiphoton resonance excitation of the excited states.

\section{ACKNOWLEDGMENTS}

This work was supported by the Chemical Sciences, Geosciences, and Biosciences Division of the Office of Basic Energy Sciences, Office of Sciences, US Department of Energy. F.-1.L. was partially supported by the National Basic Research Program of China (973 Program) No. 2010CB923102, Special Prophase Project on the National Basic Research Program of China (Grant No.2011CB311807), and the National Nature Science Foundation of China (Grant No. 11074199).
[1] H. S. Margolis et al., Science 306, 1355 (2004).

[2] A. Marian, M. C. Stowe, D. Felinto, and J. Ye, Phys. Rev. Lett. 95, 023001 (2005).

[3] J. E. Stalnaker, Y. LeCoq, T. M. Fortier, S. A. Diddams, C. W. Oates, and L. Hollberg, Phys. Rev. A 75, 040502 (2007).

[4] R. Holzwarth, T. Udem, T. W. Hansch, J. C. Knight, W. J. Wadsworth, and P. S. J. Russell, Phys. Rev. Lett. 85, 2264 (2000).

[5] M. Niering et al., Phys. Rev. Lett. 84, 5496 (2000).

[6] M. Takamoto, F. L. Hong, R. Higashi, and H. Katori, Nature (London) 435, 321 (2005).

[7] S. A. Diddams et al., Science 293, 825 (2001).

[8] E. Peik and Chr. Tamm, Europhys. Lett. 61, 181 (2003).

[9] W. G. Rellergert, D. DeMille, R. R. Greco, M. P. Hehlen, J. R. Torgerson, and E. R. Hudson, Phys. Rev. Lett. 104, 200802 (2010).

[10] C. J. Campbell, A. G. Radnaev, and A. Kuzmich, Phys. Rev. Lett. 106, 223001 (2011)

[11] V. Gerginov, C. E. Tanner, S. A. Diddams, A. Bartels, and L. Hollberg, Opt. Lett. 30, 1734 (2005).
[12] M. Fischer et al., Phys. Rev. Lett. 92, 230802 (2004).

[13] V. A. Dzuba and V. V. Flambaum, Phys. Rev. A 61, 034502 (2000).

[14] M. Herrmann et al., Phys. Rev. A 79, 052505 (2009).

[15] D. Z. Kandula, C. Gohle, T. J. Pinkert, W. Ubachs, and K. S. E. Eikema, Phys. Rev. Lett. 105, 063001 (2010).

[16] H. Mabuchi, J. Ye, and H. J. Kimble, Appl. Phys. B 68, 1095 (1999).

[17] E. E. Eyler et al., Eur. Phys. J. D 48, 43 (2008).

[18] M. J. Thorpe, K. D. Moll, R. J. Jones, B. Safdi, and J. Ye, Science 311, 1595 (2006).

[19] S. A. Diddams, L. Hollberg, and V. Mbele, Nature (London) 445, 627 (2007).

[20] Th. Udem, R. Holzwarth, and T. W. Hänsch, Nature (London) 416, 233 (2002).

[21] S. T. Cundiff and J. Ye, Rev. Mod. Phys. 75, 325 (2003).

[22] M. Hentschel et al., Nature (London) 414, 509 (2001).

[23] M. Drescher et al., Science 291, 1923 (2001). 
[24] A. Apolonski, A. Poppe, G. Tempea, C. Spielmann, T. Udem, R. Holzwarth, T. W. Hansch, and F. Krausz, Phys. Rev. Lett. 85, 740 (2000).

[25] D. J. Jones et al., Science 288, 635 (2000).

[26] C. Benko et al., Opt. Lett. 37, 2196 (2012).

[27] J. F. Hergott et al., Opt. Express 19, 19935 (2011).

[28] A. Marian, M. C. Stowe, J. R. Lawall, D. Felinto, and J. Ye, Science 306, 2063 (2004).

[29] M. C. Stowe, F. C. Cruz, A. Marian, and J. Ye, Phys. Rev. Lett. 96, 153001 (2006).

[30] A. Pe'er, E. A. Shapiro, M. C. Stowe, M. Shapiro, and J. Ye, Phys. Rev. Lett. 98, 113004 (2007).

[31] A. de Bohan, P. Antoine, D. B. Milošević, and B. Piraux, Phys. Rev. Lett. 81, 1837 (1998).

[32] R. Kienberger et al., Nature (London) 427, 817 (2004).

[33] G. Sansone et al., Science 314, 443 (2006).

[34] C. Gohle et al., Nature (London) 436, 234 (2005).

[35] R. J. Jones, K. D. Moll, M. J. Thorpe, and J. Ye, Phys. Rev. Lett. 94, 193201 (2005).

[36] J. J. Carrera, S.-K. Son, and S.-I. Chu, Phys. Rev. A 77, 031401(R) (2008).
[37] J. J. Carrera and S.-I. Chu, Phys. Rev. A 79, 063410 (2009).

[38] D. C. Yost et al., Nat. Phys. 5, 815 (2009).

[39] A. Cingöz et al., Nature (London) 482, 68 (2012).

[40] D. Z. Kandula, C. Gohle, T. J. Pinkert, W. Ubachs, and K. S. E. Eikema, Phys. Rev. A 84, 062512 (2011).

[41] A. K. Mills, T. J. Hammond, M. H. C. Lam, and D. J. Jones, J. Phys. B 45, 142001 (2012).

[42] N. F. Ramsey, Phys. Rev. 76, 996 (1949).

[43] S. Witte, R. Th. Zinkstok, W. Ubachs, W. Hogervorst, and K. S. E. Eikema, Science 307, 400 (2005).

[44] S.-K. Son and S.-I. Chu, Phys. Rev. A 77, 063406 (2008).

[45] S. I. Chu and D. A. Telnov, Phys. Rep. 390, 1 (2004).

[46] T. S. Ho, S. I. Chu, and J. V. Tietz, Chem. Phys. Lett. 96, 464 (1983).

[47] T. S. Ho and S. I. Chu, J. Phys. B 17, 2101 (1984).

[48] T.-S. Ho and S.-I. Chu, Phys. Rev. A 31, 659 (1985).

[49] T.-S. Ho and S.-I. Chu, Phys. Rev. A 32, 377 (1985).

[50] S. I. Chu and W. P. Reinhardt, Phys. Rev. Lett. 39, 1195 (1977).

[51] G. Yao and S. I. Chu, Chem. Phys. Lett. 204, 381 (1993). 